\title{
Social determinants of health and adult influenza vaccination: a nationwide claims analysis
}

\author{
Justin Gatwood, PhD, MPH; Sujith Ramachandran, PhD; Sohul A Shuvo, MS; Michael Behal, PharmD; \\ Tracy Hagemann, PharmD; Kenneth C Hohmeier, PharmD; and Chi-Yang Chiu, PhD
}

\section{What is already known about this subject}

- Regular vaccination against seasonal influenza continues to miss stated national goals among the general public.

- Social determinants of health are being increasingly investigated as significant contributing factors to the uptake of certain health services, including vaccines.
What this study adds

- Factors not related to health care are playing an increasingly important role in influencing ongoing seasonal influenza vaccination, the effects of which may differ between working-age and elderly adults.

- Given the observed regional variation in influenza vaccine adherence, results suggest that more granular assessment of local influencers on vaccinations are warranted so that community-level factors may be identified and managed.

\section{Author affiliations}

Justin Gatwood, PhD, MPH; Tracy Hagemann, PharmD; and Kenneth C Hohmeier, PharmD, University of Tennessee Health Science Center College of Pharmacy, Nashville. Sujith Ramachandran, PhD, University of Mississippi School of Pharmacy, Oxford. Sohul A Shuvo, MS, College of Graduate Health Sciences, and Chi-Yang Chiu, PhD, College of Medicine, University of Tennessee Health Science Center, Memphis. Michael Behal, PharmD, University of Kentucky HealthCare, Lexington.

AUTHOR CORRESPONDENCE: Justin Gatwood, 615.532.3403; jgatwood@uthsc.edu

J Manag Care Spec Pharm. 2022;28(2):196-205

Copyright $@ 2022$, Academy of Managed Care Pharmacy. All rights reserved.

\section{ABSTRACT}

BACKGROUND: The health and economic benefits of the annual influenza vaccine are well documented, yet vaccination rates in the United States missed the Healthy People 2020 goal and remain a focus of Healthy People 2030 efforts. By identifying underlying reasons for low annual influenza vaccination, social elements that need targeting may be identified and could guide future interventions or policy development to achieve vaccination goals and improve overall public health.
OBJECTIVE: To determine the influence of certain social determinants of health on adherence to annual influenza vaccination in American adults.

METHODS: This was a retrospective cohort analysis using data from IBM MarketScan Commercial Claims and Encounters Database and national Medicare 5\% sample data from 2013 to 2016. Study eligibility criteria included adults (aged 18 years and older) who were continuously enrolled for 3 influenza seasons between 2013 and 2016. Receipt of the influenza vaccine was counted over 3 consecutive influenza seasons, and select social determinants were extracted from publicly available sources. Patient characteristics, health resource utilization, and selected social determinants of health were included in bivariate and multivariate logistic regression analyses to determine their association with annual influenza vaccination.

RESULTS: 6,694,571 adults across employersponsored and Medicare coverage groups were analyzed, of which $14.7 \%$ of Medicare-enrolled adults and $9.2 \%$ of commercially enrolled adults were vaccinated in all 3 seasons. Higher proportions of vaccine adherence (ie, all 3 seasons) were 
observed among females ( $9.6 \%$ vs $8.7 \%$ [commercial], $15.0 \%$ vs $14.4 \%$ [Medicare]), the immunocompromised ( $11.8 \%$ vs $8.3 \%$ [commercial], $15.9 \%$ vs $13.6 \%$ [Medicare]), rural residents (10.5\% vs $9.0 \%$ [commercial], $15.4 \%$ vs $14.6 \%$ [Medicare]; all $P<0.0001$ ), and those enrolled in a high-deductible health plan (10.3\%). Multivariable logistic regression models indicated that the odds of vaccine adherence tended to be higher in areas of higher poverty $(\mathrm{OR}=1.012 ; 95 \% \mathrm{Cl}=1.01-1.02$ [commercial], $\mathrm{OR}=1.01 ; 95 \% \mathrm{Cl}=1.01-1.01$ [Medicare]) yet lower in areas with higher proportions of Democratic voters $(\mathrm{OR}=0.998$; $95 \% \mathrm{Cl}=0.998-0.998$ [commercial], $\mathrm{OR}=0.996 ; 95 \% \mathrm{Cl}=0.996-0.997$ [Medicare]). Among commercially insured adults, the odds of vaccine adherence were higher in areas of higher health literacy ( $\mathrm{OR}=1.036 ; 95 \% \mathrm{Cl}=1.036-1.037)$, but this effect was not observed among Medicare members. Conversely, the odds of vaccine adherence increased as the proportion of those residing in areas of limited Internet access increased ( $\mathrm{OR}=1.007 ; 95 \% \mathrm{Cl}=1.004-1.010)$ among Medicare members only.

CONCLUSIONS: Key social determinants of health are important factors of vaccine adherence and can guide policy and intervention efforts toward addressing potential hesitancy. A deeper assessment of other contributing social factors is needed in seasonal influenza and other vaccines to better interpret the vaccine-seeking behaviors of adults.

Influenza vaccinations have contributed to a significant decrease in hospitalizations, emergency department visits, outpatient medical visits, and respiratory- and circulatory-related deaths in the United States. ${ }^{1}$ In addition to benefitting those who receive the vaccine, high influenza vaccination rates contribute to herd immunity, offering protection to individuals who opt not or are unable to receive the vaccine. ${ }^{2}$ It is estimated that the total US economic burden associated with influenza is over \$5.8 billion annually. ${ }^{3}$ Although research has demonstrated the health and economic benefits of the annual influenza vaccine, US vaccination rates fell below the Healthy People 2020 goal of $80 \%$, having attained only $45.3 \%$ for the $2018-2019$ influenza season, and improving these rates was included in the Healthy People 2030 goals. ${ }^{4-6}$ Although this represents an increase from the 2017-2018 influenza season rate of $37.1 \%$, significant room for improvement still exists. ${ }^{7}$

A number of potential barriers to influenza vaccination have been identified, with most research centering on access issues, personal beliefs, and awareness. To better understand and combat potential barriers, the SAGE Working Group on Vaccine Hesitancy comprehensively assessed reasons that individuals may delay or refuse vaccinations. ${ }^{8}$ Data collected from the WHO-UNICEF Joint Reporting Form determined that concern for side effects, lack of knowledge regarding risks of vaccine-preventable diseases, and antivaccination reports across media platforms were the main contributors to vaccine hesitancy. ${ }^{9}$ The working group concluded that vaccine hesitancy trends can vary significantly from year to year and would benefit from ongoing monitoring. ${ }^{8}$

Identifying the sociocultural context of individually held beliefs may help decrease the prevalence of vaccine hesitancy. Broadly defined by the World Health Organization (WHO), social determinants of health (SDoH) are "the context in which an individual is born, matures, and ages," and these factors play a role in determining health inequality. ${ }^{10,11}$ The use of $\mathrm{SDoH}$ as an approach to research is endorsed by WHO as a method for investigating the sociocultural issues in adult vaccination. ${ }^{12}$

Although previous studies have explored certain reasons for patients delaying or refusing vaccines, it should be noted that factors will likely vary across different periods of time, regions, and vaccines. ${ }^{9,13}$ Therefore, identified factors are likely not generalizable to all vaccine types and should be studied within specific contexts. Moreover, $\mathrm{SDoH}$ and vaccine hesitancy research has historically focused on the pediatric population, as evidenced by frameworks developed to tackle this issue globally. ${ }^{14}$ However, recent research examined $\mathrm{SDoH}$ and influenza vaccine hesitancy within adult populations, determining that trust in government, political partisanship, and fear of infection correlated with influenza vaccination willingness in adults across the United States. ${ }^{15}$

Trust in the influenza vaccine has also been identified as a common barrier, with 1 study showing that older age, higher household income, and more liberal political views correlated with higher trust in the influenza vaccine. ${ }^{16}$ Among individuals aged 65 years and older, sociocultural aspects affecting influenza vaccine acceptance include perceived susceptibility, previous experiences, and personal beliefs about the vaccine and disease. ${ }^{17}$ A large systematic review identified limited interaction with health care personnel and fewer cues to action (ie, recommendations from a health care provider or even a relative to receive the vaccine) as being correlated with influenza vaccine hesitancy. ${ }^{18}$ These initial investigations provide valuable guidance on what drives adult vaccine hesitancy, but a range of factors remain to be examined in order to better interpret adult vaccine-seeking behaviors.

Noting a gap in our understanding of vaccine hesitancy in adults, this study aimed to determine the influence of certain $\mathrm{SDoH}$ on annual influenza vaccination behaviors in American adults. By identifying underlying reasons for nonadherence to annual influenza vaccination, social elements that need targeting may be identified and could 
guide future interventions or policy development in order to achieve Healthy People 2030 vaccination goals and improve overall public health.

\section{Methods}

Data for this study were derived from the IBM MarketScan Commercial Claims and Encounters databases and a national Medicare 5\% sample data for years 2013-2016. The MarketScan databases are nationwide convenience samples of the insurance enrollment and health care resource use of millions of Americans with private insurance, and the data are deidentified and fully compliant with the Health Insurance Portability and Accountability Act of 1996 (HIPAA). The use of these data was determined to be nonhuman subjects research by the Institutional Review Board (IRB) at the University of Tennessee Health Science Center. Medicare claims data were licensed from the Centers for Medicare \& Medicaid Services (DUA\#RSCH-2019-53502) and approved for use by the University of Mississippi IRB.

The main outcome of interest was receipt of the seasonal influenza vaccine over 3 consecutive influenza seasons, which were defined as running from July 1 through June 30 of the following year and created 3 distinct observation periods spanning 2013-2014, 2014-2015, and 2015-2016. To be included in the analysis, patients needed to be aged 18 years or older and have continuous enrollment over the influenza seasons observed. Receipt of the influenza vaccine in each season was determined using outpatient encounters and pharmacy claims (National Drug Code numbers and procedure codes are listed in Supplementary Table 1, available in online article). Vaccine coverage among the eligible population during each observation period was estimated, and those receiving an influenza vaccine in each of the 3 years were deemed adherent.

The main predictors of the analyses were $\mathrm{SDoH}$ factors derived from multiple, publicly available data sources. The WHO suggests that hesitancy toward vaccines is prominent and that a range of factors contribute to vaccine-seeking behaviors. These are described by the Vaccine Hesitancy Determinants Matrix, which characterizes influencers as contextual (eg, media and influential leaders); individual/ group (eg, personal experiences, heuristics, and social norms); and those vaccine-specific (eg, administration and cost). ${ }^{8}$ Most studies that have examined these factors have focused on the decisions made regarding childhood vaccinations, with little research devoted to how these influencers may affect decisions by adults.

Consideration was also made for elements outlined by the SDoH framework, the 5 pillars of which include health care access and quality, education access and quality, social and community context, economic stability, and neighborhood and built environment. ${ }^{19}$ Guided by overlaps between these frameworks, we identified several sources of data that may serve as suitable proxies for several contextual and individual/group influences of the hesitancy matrix. Such data were derived from the University of North Carolina (health literacy), county-level election data compiled by MIT for the 2012 presidential election (voting records by political party), and the American Community Survey (poverty, Internet, and computer ownership). ${ }^{20-22}$ Consequently, several pillars of the $\mathrm{SDoH}$ framework were represented by these proxies for elements of the Determinants Matrix: health care access and quality (health literacy), neighborhood and built environment (Internet access and computer ownership), economic stability (poverty), and social and community context (voting records).

Because the geospatial elements of the data were limited to the metropolitan statistical area (MSA) level, $\mathrm{SDoH}$ values were averaged across each MSA. Such geographic proxies have been previously used in research to identify the burden of SDoH on health outcomes, including our previous analyses on pneumococcal and herpes zoster vaccinations. ${ }^{23-26}$ As proxies for directly observed SDoH values, these data facilitate our interpretation of their effect on eventual vaccination related to patient understanding of health information, conservative vs progressive political leanings, local socioeconomics, and access to information.

In addition to the $\mathrm{SDoH}$ variables, demographic and clinical characteristics available in all datasets included age, sex, residential region and MSA of residence; health plan type was also included for commercially insured patients. Additional factors derived or calculated from claims data included rural residence; immunocompetency (diagnosis codes are listed in Supplementary Table 2, available in online article); and health resource utilization (number of outpatient visits, inpatient admissions, and pharmacy visits).

Differences in demographics and clinical characteristics across vaccine adherence categories were assessed using t-tests and chi-square tests for continuous and categorical variables, respectively. Chi-square tests determined differences in the proportion of adults vaccinated in all 3 years within categorical variables. Logistic regression determined the odds of getting vaccinated in all 3 seasons, while controlling for available demographic and $\mathrm{SDoH}$ variables.

Two different statistical models were run within each database population (MarketScan and Medicare). First, a population density model was constructed, which included all eligible patients and examined the influence of rurality 


\begin{tabular}{|c|c|c|}
\hline Characteristic & $\begin{array}{c}\text { Commercial } \\
\mathbf{n}(\%)\end{array}$ & $\begin{array}{c}\text { Medicare } \\
\text { fee-for-service } \\
n(\%)\end{array}$ \\
\hline Total & $6,089,487$ & 605,084 \\
\hline Age, years, mean (SD) & $44.1 \quad(11.6)$ & $(7.2)$ \\
\hline $18-24$ & $492,368 \quad(8.1)$ & \\
\hline $25-34$ & $823,622 \quad(13.5)$ & \\
\hline $35-44$ & $1,418,404 \quad(23.3)$ & \\
\hline $45-54$ & $2,008,912 \quad(33.0)$ & \\
\hline $55-64$ & $1,346,181 \quad(22.1)$ & \\
\hline $65-74$ & & $346,263 \quad(57.2)$ \\
\hline $75+$ & & $258,821 \quad(42.8)$ \\
\hline Male & $2,498,307 \quad(41.0)$ & $233,487 \quad(38.6)$ \\
\hline \multicolumn{3}{|l|}{ Region } \\
\hline Northeast & $1,129,361 \quad(18.6)$ & $122,196 \quad(20.6)$ \\
\hline Midwest & $1,269,158 \quad(20.7)$ & $145,522 \quad(24.5)$ \\
\hline South & $2,763,947 \quad(45.4)$ & $216,149 \quad(36.5)$ \\
\hline West & $933,275 \quad(15.3)$ & $109,127 \quad(18.4)$ \\
\hline Relocated & $503,821 \quad(8.3)$ & \\
\hline Rural & $856,186 \quad(14.1)$ & $67,095 \quad(11.1)$ \\
\hline \multicolumn{3}{|l|}{ Plan type } \\
\hline Comprehensive & $141,897 \quad(2.4)$ & \\
\hline $\mathrm{HMO}$ & $815,334 \quad(13.5)$ & \\
\hline POS & $542,011 \quad(9.0)$ & \\
\hline PPO/EPO & $3,763,641 \quad(62.3)$ & \\
\hline CHDP/HDHP & $780,072 \quad(12.9)$ & \\
\hline Immunocompromised & $1,654,087 \quad(27.2)$ & $315,946 \quad(52.2)$ \\
\hline \multicolumn{3}{|l|}{ Resource utilization ${ }^{a}$} \\
\hline Inpatient admissions & $0.3 \quad(0.79)$ & $(1.2)$ \\
\hline Outpatient visits & $7.1 \quad(12.7)$ & $95.9 \quad(75.9)$ \\
\hline ED visits & $0.8 \quad(2.1)$ & $(5.0)$ \\
\hline Prescription fills & $43.0 \quad(43.8)$ & $110.0(100.8)$ \\
\hline \multicolumn{3}{|c|}{$\begin{array}{l}\text { Note: Missing (commercial/Medicare fee-for-service: region }(N=3,746 / 12,090 \\
\text { plan type }(N=46,532) . \\
\text { aResource utilization measures over entire observation period. } \\
C H D P=\text { consumer-directed health plan; } E D=\text { emergency department; } \\
\text { EPO= exclusive provider organization; } H D H P=\text { high-deductible health } \\
\text { plan; } H M O=\text { health maintenance organization; } P O S=\text { point of service; } \\
P P O=\text { preferred provider organization. }\end{array}$} \\
\hline
\end{tabular}

of individual residence on vaccine adherence while controlling for demographic and clinical characteristics. Second, a social determinants model included the same predictor variables as the first model but substituted $\mathrm{SDoH}$ predictors for population density and only included those individuals who resided in MSAs (ie, no rural patients) due to an inability to attribute $\mathrm{SDoH}$ values to non-MSA residents. An alpha of 0.05 was set a priori to determine statistical significance, and SAS version 9.4 (SAS Institute) was used for all analyses.

\section{Results}

\section{PATIENT POPULATION}

A total of $6,694,571$ adults across both datasets met inclusion criteria and were analyzed (selection listed in Supplementary Table 2). Both Medicare and commercial cohorts had a higher proportion of female patients $(61.4 \%$ and $59.0 \%$, respectively), were predominantly not immunocompromised, and had a larger share of adults residing in the southern region of the country (Table 1).

\section{INFLUENZA VACCINATION}

In each season analyzed, a minority of adults were vaccinated against seasonal influenza, irrespective of cohort. For commercially insured patients, slight increases in the proportions vaccinated were realized in each year, yet noticeable decreases were observed among Medicare patients (Figure 1). Over the course of the 3 seasons, nearly two-thirds of commercially insured adults (63.9\%) and approximately 2 out of 5 Medicare enrollees (41.1\%) were never vaccinated. A larger share of Medicare enrollees was adherent to the influenza vaccine in all 3 seasons: $14.7 \%$ vs $9.2 \%$ (commercial).

Among commercially insured patients, the proportion adherent tended to increase with age, peaking at $14.6 \%$ of those aged 55-64 years, and enrollees of a consumer-driven (CDHP) or high-deductible (HDHP) health plan $(10.3 \%$, $\mathrm{P}<0.0001$ compared with other plan types) were also more likely to have been vaccinated in all 3 seasons.

Irrespective of cohort, other groups with higher proportions adherent (all $\mathrm{P}<0.0001)$ included females $(9.6 \%$ vs $8.7 \%$ [commercial], $15.0 \%$ vs $14.4 \%$ [Medicare]), residents of rural areas $(10.5 \%$ vs $9.0 \%$ [commercial], $15.4 \%$ vs $14.6 \%$ [Medicare]), immunocompromised adults (11.8\% vs $8.3 \%$ [commercial], $15.9 \%$ vs $13.6 \%$ [Medicare]), and those who did not relocate (eg, change in MSA or rural to urban) during the observation period (9.3\% vs $8.6 \%$ [commercial], $14.9 \%$ vs $11.6 \%$ [Medicare]).

\section{PREDICTORS OF INFLUENZA VACCINE ADHERENCE}

Multiple logistic regression models were fit for each cohort to predict the odds of having been vaccinated in all 3 influenza seasons. Results, in terms of direction and magnitude, were generally similar between the commercial and Medicare cohorts (Table 2). The odds of adherence tended 


\section{FIGURE 1 Proportion of Adults Vaccinated in Each Influenza Season, 2013-2016}

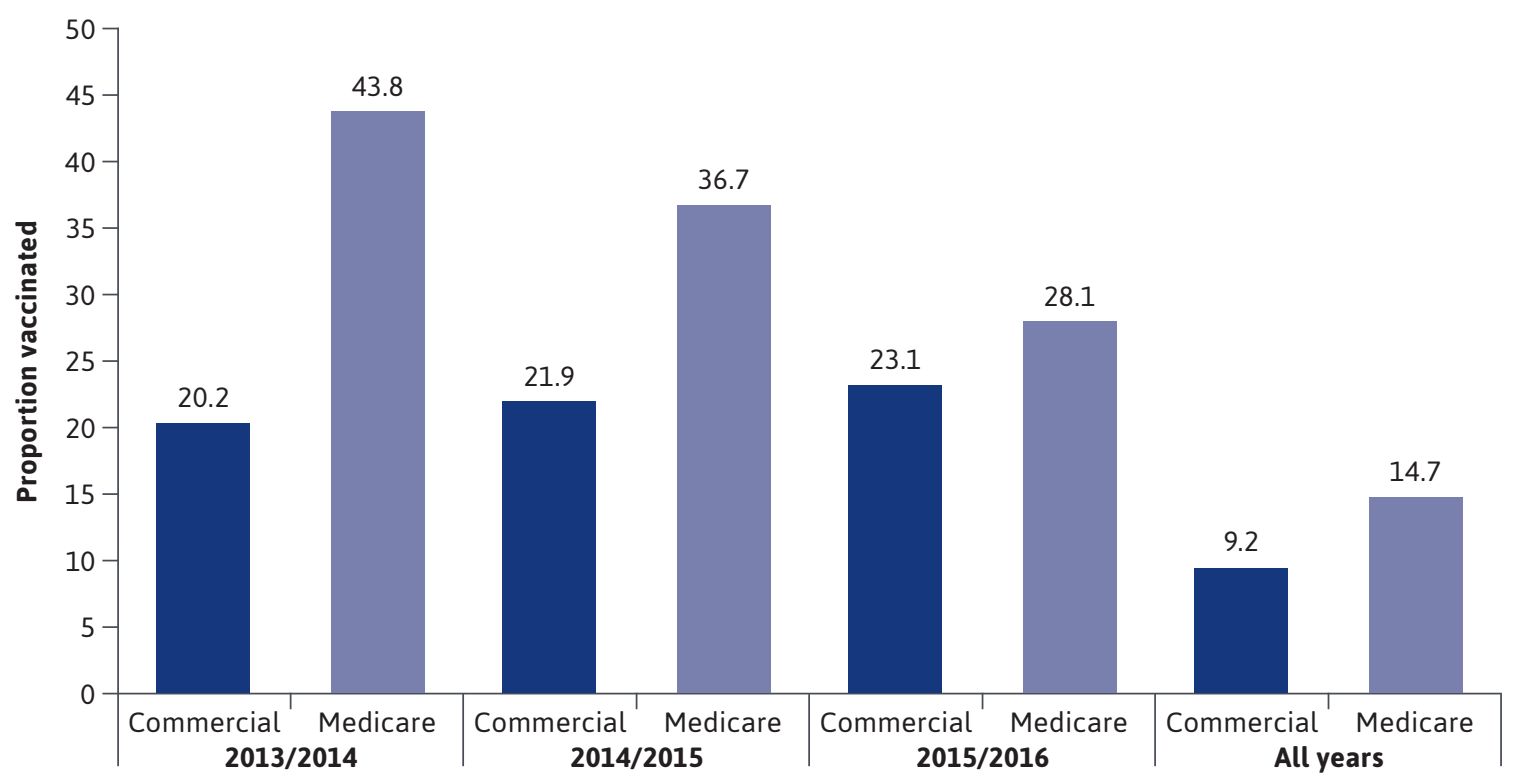

to increase with age, were lower among males, and less likely among the immunocompetent. Additionally, those not relocating outside their baseline (2013) MSA were more likely to vaccinate in all 3 seasons, irrespective of cohort (commercial odds ratio $[\mathrm{OR}]=1.08 ; 95 \% \mathrm{CI}=1.067-1.089$; Medicare $\mathrm{OR}=1.31 ; 95 \% \mathrm{CI}=1.265-1.362$ ).

In both cohorts, those with more hospital admissions or higher emergency department use were less likely to be vaccinated in all 3 seasons, whereas those with a higher number of outpatient or pharmacy visits were more likely to be adherent to the vaccine, although the magnitude was marginal. Changes in plan types over the observation period had differing effects between groups because commercially insured adults who did not change plans were less likely to be adherent $(\mathrm{OR}=0.90 ; 95 \% \mathrm{CI}=0.897-0.910)$, whereas Medicare enrollees were more likely to vaccinate in all 3 seasons $(\mathrm{OR}=1.14 ; 95 \% \mathrm{CI}=1.047-1.234)$.

In the separate models, similar differences in the direction of odds were observed by population density in each cohort: urban residents with commercial insurance were less likely to adhere to the vaccine $(\mathrm{OR}=0.87 ; 95 \% \mathrm{CI}=0.867-$ 0.881), whereas urban Medicare members were more likely to adhere $(\mathrm{OR}=1.12 ; 95 \% \mathrm{CI}=1.098-1.150)$.

In terms of social determinants, results were similar across cohorts. Specifically, the odds of vaccine adherence tended to be higher in areas of higher poverty $(\mathrm{OR}=1.012$; 95\% CI=1.01-1.02 [commercial], OR=1.01; 95\% CI=1.01-1.01
[Medicare]) yet lower in areas with higher proportions of Democratic voters $(\mathrm{OR}=0.998 ; 95 \% \mathrm{CI}=0.998-0.998$ [commercial], $\mathrm{OR}=0.996$ 95\% CI=0.996-0.997 [Medicare]).

Among commercially insured adults, the odds of vaccine adherence were higher among areas of higher health literacy $(\mathrm{OR}=1.036 ; 95 \% \mathrm{CI}=1.036-1.037)$, but this effect was not observed among Medicare members $(\mathrm{OR}=1.001$; 95\% CI $=0.996-1.003)$. Conversely, the odds of vaccine adherence increased as the proportion of those residing in areas of limited Internet access increased $(\mathrm{OR}=1.007 ; 95 \%$ $\mathrm{CI}=1.004-1.010)$ among Medicare members only.

\section{GEOGRAPHIC VARIATION IN INFLUENZA VACCINE ADHERENCE}

Unadjusted proportions of influenza vaccine adherence were well distributed across the country in both cohorts. Among commercially insured adults, each region of the country had several states represented in the upper quartile of completion percentages, with the South having the highest individual count yet also a high proportion of states in the lower 2 quartiles (Figure 2). Tennessee (16.6\%) and Kentucky (15.7\%) had the highest percentage of adherence among all states. Logistic regression results (using western states as the reference) showed no clear trend in geographic variation among the commercial cohort and with only slight differences in the unadjusted percentage of adherence across the region. 


\section{TABLE 2}

Odds of Influenza Vaccination Adherence in Adults by Payer

\begin{tabular}{|c|c|c|c|c|}
\hline \multirow[b]{2}{*}{ Characteristic } & \multicolumn{2}{|c|}{ Commerciala $^{a}$} & \multicolumn{2}{|c|}{ Medicare fee-for-service $^{b}$} \\
\hline & OR (95\% CI) & $P$ value & OR $(95 \% \mathrm{CI})$ & $P$ value \\
\hline \multicolumn{5}{|l|}{ Age, years } \\
\hline $18-24$ & $0.29(0.284-0.294)$ & $<0.0001$ & & \\
\hline $25-34$ & $0.49(0.482-0.492)$ & $<0.0001$ & & \\
\hline $35-44$ & $0.53(0.526-0.535)$ & $<0.0001$ & & \\
\hline $45-54$ & $0.66(0.657-0.666)$ & $<0.0001$ & & \\
\hline $55-64$ & \multicolumn{2}{|l|}{ ref } & & \\
\hline $65-74$ & & & $0.78 \quad(0.770-0.793)$ & $<0.0001$ \\
\hline $75+$ & & & \multicolumn{2}{|l|}{ ref } \\
\hline \multicolumn{5}{|l|}{ Sex } \\
\hline Male & $0.895(0.890-0.901)$ & $<0.0001$ & $0.98 \quad(0.965-0.994)$ & 0.0064 \\
\hline Female & \multicolumn{2}{|l|}{ ref } & \multicolumn{2}{|l|}{ ref } \\
\hline \multicolumn{5}{|l|}{ Region } \\
\hline Northeast & $1.06(1.046-1.066)$ & $<0.0001$ & 1.14 (1.110-1.163) & $<0.0001$ \\
\hline Midwest & $1.01(1.002-1.022)$ & 0.2355 & $1.29(1.256-1.316)$ & $<0.0001$ \\
\hline South & $0.97(0.960-0.976)$ & $<0.0001$ & $1.12(1.095-1.144)$ & $<0.0001$ \\
\hline West & \multicolumn{2}{|l|}{ ref } & \multicolumn{2}{|l|}{ ref } \\
\hline \multicolumn{5}{|l|}{ Relocated } \\
\hline Yes & \multicolumn{2}{|l|}{ ref } & \multicolumn{2}{|l|}{ ref } \\
\hline No & 1.08 (1.067-1.089) & $<0.0001$ & $1.31(1.265-1.362)$ & $<0.0001$ \\
\hline \multicolumn{5}{|l|}{ Plan type } \\
\hline Comprehensive & $0.57(0.558-0.582)$ & $<0.0001$ & & \\
\hline HMO & $0.88(0.873-0.892)$ & $<0.0001$ & & \\
\hline POS & $0.90(0.886-0.907)$ & $<0.0001$ & & \\
\hline PPO/EPO & $0.82(0.812-0.826)$ & 0.8767 & & \\
\hline CHDP/HDHP & \multicolumn{2}{|l|}{ ref } & & \\
\hline
\end{tabular}

continued on next page

In contrast, the odds of being adherent among Medicare members were consistently higher in the Northeast, Midwest, and South compared with the West-comparisons that are apparent in the mapped distribution of unadjusted proportions (Figure 3) wherein a high number of western states are in the lowest quartile. Among the Medicare cohort, Hawaii (21.2\%), North Carolina (20.6\%), and Arkansas (20.5\%) had the highest proportion of residents adherent to the influenza vaccine over the 3-season observation period.

\section{Discussion}

This study highlights a pervasive issue across the US health care system: poor influenza vaccination among adults. Although slight increases in the proportion vaccinated were observed in commercially insured adults, these figures are suboptimal based on Centers for Disease Control and Prevention (CDC) guidelines, and relatively few adults are consistently vaccinating against seasonal influenza. Perhaps more alarming was the observed year-over-year decline in the proportions vaccinated among Medicare members. While older adults achieved a higher proportion vaccinated in all 3 years, fewer than 1 in 5 reached such a threshold, which paints a somewhat dismal picture of the US ability to widely vaccinate against seasonal influenza. Arguably, there are a number of factors that influence initial and subsequent decisions to become vaccinated (eg, vaccine effectiveness, cost, access, and previous vaccination experiences), but the relatively low rates by which adults appear to be seeking influenza vaccination suggest that more must be done if target immunization goals are to be achieved.

While the proportions vaccinated in the Medicare population are alarming, those in the commercial population are also cause for concern. Despite the marginal, year-over-year increases in the proportion vaccinated, the proportion of those aged 18-64 years being consistently vaccinated remained below $10 \%$, which is substantially below what Healthy People 2020 had originally established as a goal for this portion of the population, as well as the updated Healthy People 2030 goal of $70 \%{ }^{27,28}$ Although assessment of vaccinating against seasonal influenza in this population (ie, working-age adults) has found this preventive measure to not be generally cost saving, it remains a mechanism recommended by the CDC's Advisory Committee on Immunization Practices as a means to prevent millions of cases of illness, while simultaneously limiting direct and indirect economic impacts., ${ }^{1,29}$

Although workplace influenza vaccination has been used by firms as an efficient means to increase vaccination rates (and, thus, reduce the odds 


\section{TABLE 2 Odds of Influenza Vaccination Adherence} in Adults by Payer (continued)

\begin{tabular}{|c|c|c|c|c|}
\hline \multirow[b]{2}{*}{ Characteristic } & \multicolumn{2}{|c|}{ Commercial $^{a}$} & \multicolumn{2}{|c|}{ Medicare fee-for-service ${ }^{b}$} \\
\hline & OR $(95 \% \mathrm{CI})$ & $P$ value & OR $(95 \% \mathrm{CI})$ & $P$ value \\
\hline \multicolumn{5}{|l|}{ Plan change } \\
\hline Yes & \multicolumn{2}{|l|}{ ref } & \multicolumn{2}{|l|}{ ref } \\
\hline No & $0.90 \quad(0.897-0.910)$ & $<0.0001$ & $(1.047-1.234)$ & 0.0022 \\
\hline \multicolumn{5}{|l|}{ Immunity status } \\
\hline Immunocompetent & $0.83 \quad(0.826-0.836)$ & $<0.0001$ & $(0.907-0.936)$ & $<0.0001$ \\
\hline Immunocompromised & \multicolumn{2}{|l|}{ ref } & \multicolumn{2}{|l|}{ ref } \\
\hline \multicolumn{5}{|l|}{ Resource utilization } \\
\hline Inpatient admission & $0.92 \quad(0.917-0.925)$ & $<0.0001$ & $0.88 \quad(0.860-0.895)$ & $<0.0001$ \\
\hline Outpatient visits & $1.002(1.002-1.003)$ & $<0.0001$ & 1.002 (1.002-1.003) & $<0.0001$ \\
\hline ED visits & $0.928(0.927-0.930)$ & $<0.0001$ & $0.977(0.975-0.979)$ & $<0.0001$ \\
\hline Prescription fills & 1.007 (1.007-1.007) & $<0.0001$ & 1.001 (1.001-1.001) & $<0.0001$ \\
\hline \multicolumn{5}{|l|}{ Social determinants } \\
\hline Poverty & 1.015 (1.014-1.017) & $<0.0001$ & $1.01 \quad(1.005-1.012)$ & $<0.0001$ \\
\hline Health literacy & $1.036(1.036-1.037)$ & $<0.0001$ & 1.001 (0.999-1.003) & 0.3097 \\
\hline Democratic voters & $0.998(0.998-0.998)$ & $<0.0001$ & $0.996(0.996-0.997)$ & $<0.0001$ \\
\hline Internet access & 1.001 (0.999-1.003) & 0.1654 & 1.007 (1.004-1.010) & $<0.0001$ \\
\hline
\end{tabular}

aModel controlled for age, sex, geographic region, immunity status, and health resource utilization.

bModel controlled for age, sex, geographic region, immunity status, and health resource utilization. C statistics for models: 0.664 commercial, 0.593 Medicare.

$C H D P=$ consumer-directed health plan; $E D=$ emergency department; $E P O=$ exclusive provider organization; $H D H P=$ high-deductible health plan; $H M O=$ health maintenance organization; $O R=$ odds ratio; $P O S=$ point of service; $P P O=$ preferred provider organization; ref = reference .

of absenteeism), such a mechanism on its own is not sufficient to adequately immunize the working-age subgroup of the country. Other means to improve access to influenza vaccines, such as availability at community pharmacies, have contributed to higher odds of adult vaccination ${ }^{30}$; however, room for improvement in annual incidence and adherence to seasonal influenza vaccination remains.

Because community pharmacies have overtaken medical clinics as the dominant source for influenza vaccination, this may require increased outreach efforts by these access points to working-age adults. ${ }^{31}$ Considering the lower odds of vaccine adherence in younger adults as the observed association between vaccination and certain resource utilization, such efforts may require a concerted focus on healthy, younger adults who are perhaps less likely to use health care resources but could conveniently seek vaccination at a pharmacy in their community.

Although vaccination adherence was well distributed across the country, several geospatial patterns are worth noting. In general, the adjusted odds of adherence were lower in western states compared with other regions of the country, and visual evidence of this disparity is particularly prominent when examining the unadjusted proportions among Medicare members in the western half of the United States. A notable concentration of better-performing states was also observed in the southeast across commercially insured adults and Medicare members, which is reassuring, since case rates have tended to be higher in southern states even though adherence rates in those states remain underwhelming. ${ }^{32}$

Additionally, the contrasting results observed across cohorts by residential area are worthy of deeper analysis. Our findings echo what the Centers for Medicare \& Medicaid Services observed recently: higher influenza vaccine coverage in urban vs rural residents. ${ }^{33}$ However, the observation that working-age adults residing in urban areas were less likely to consistently vaccinate suggests that access alone may not be sufficient to ensure that adults seek regular, recommended, preventive treatment. Increasing the use of clinical decision support within community pharmacies and provider offices to include such measures as provider prompts and automated messages, may help to play a role in increasing rates of vaccination in urban and rural localities ${ }^{34,35}$

However, these mechanisms may not be sufficient to address the needs of patients migrating across health systems. The current results indicated that relocating during the observation period was associated with poorer adherence to seasonal influenza vaccination, suggesting that disruptions to regular access and use of health care resources may be an impediment to continued vaccination. In these circumstances, interventions at a payer level may be necessary to encourage patients to again seek vaccination in their new residential areas and then assist them with finding a convenient access point, while adjusting to receiving services in a new area. ${ }^{36}$

Commercially insured and Medicare members were more likely to receive influenza vaccine if they were 


\begin{tabular}{|c|c|}
\hline FIGURE 2 & $\begin{array}{l}\text { Geographic Variation in Proportion } \\
\text { Adherent to Influenza Vaccination } \\
\text { Among Commercially Insured Adults, } \\
\text { 2013-2016 }\end{array}$ \\
\hline
\end{tabular}

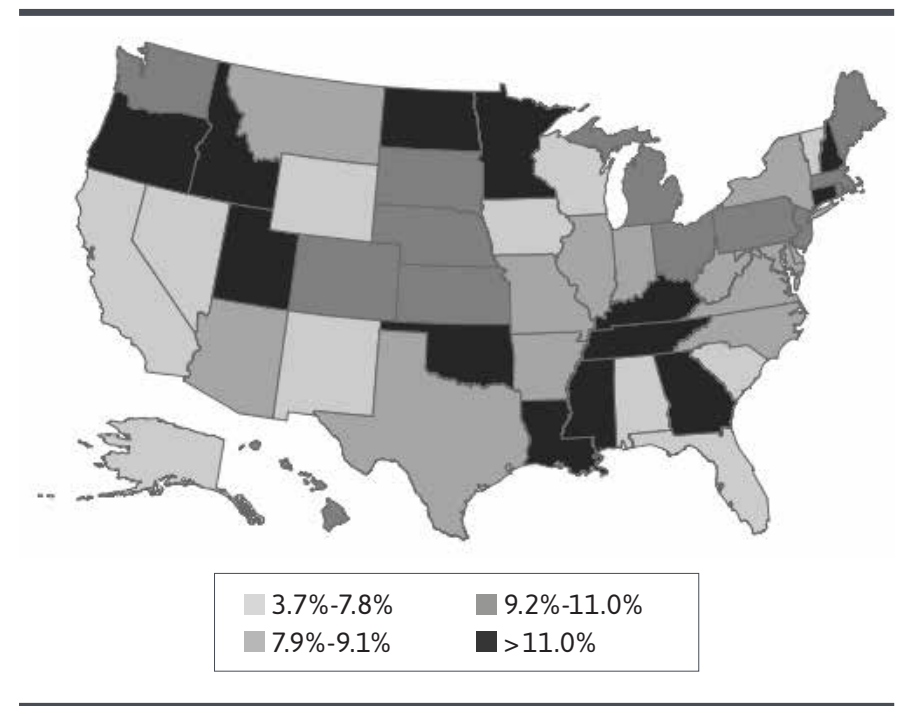

in higher-poverty areas, and this is consistent with what has been previously published. ${ }^{37,38}$ In this study, commercially insured adults who resided in high health literacy areas were more likely to consistently receive the flu vaccine compared with those adults enrolled in traditional Medicare. Also, while the role of health literacy in vaccination adherence seems to be positively influenced by risk perceptions and the likelihood of getting sick, the observed variation between types of insurance is an area that requires more investigation. ${ }^{39}$ Specifically, the increased odds of those on more consumer-directed plans (eg, CDHP and HDHP) suggest that regular vaccination may be indicative of those enrolled in these types of plans being more engaged with preventive behaviors that would reduce the chances of expensive downstream health care costs.

Interestingly, the odds of influenza vaccination increased in the Medicare population as the availability/reliability of Internet access decreased. This seems to contradict research that better informed adults are more likely to be vaccinated..$^{40}$ However, the quality of vaccination information available online, especially in social media settings, has been documented, and this has increased in the recent COVID-19 pandemic. ${ }^{41,42}$

\section{LIMITATIONS}

The results of this study should be interpreted with several limitations in mind. First, the data were based on claims
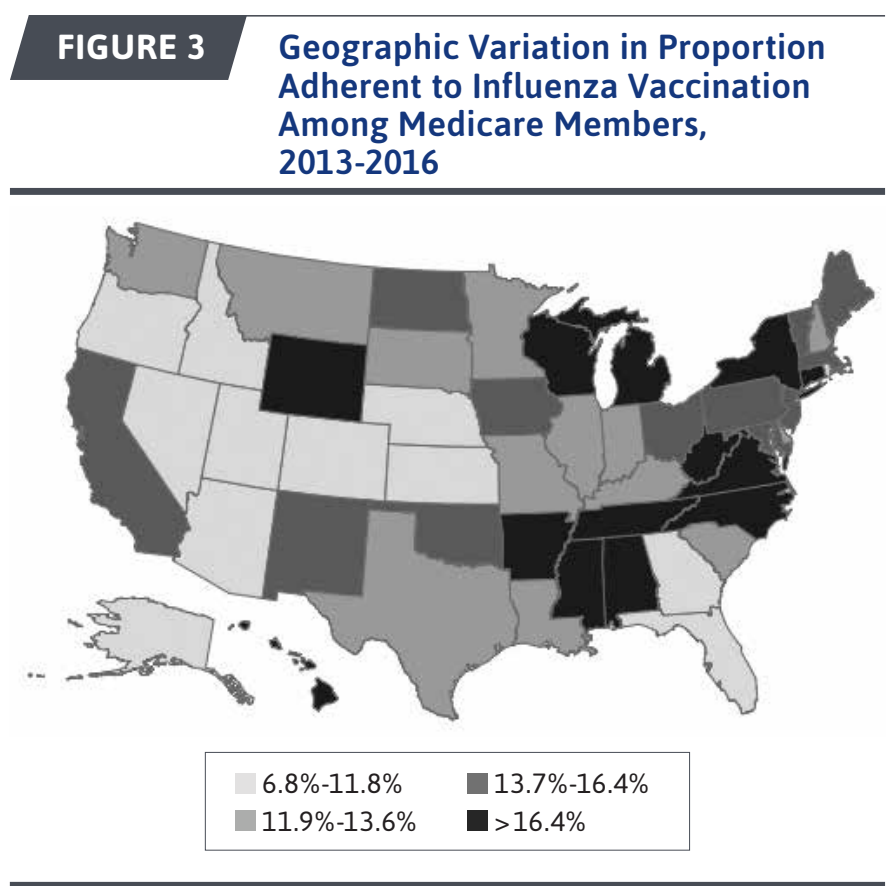

made for payment for services; therefore, vaccinations provided for which a claim was not generated would not have been observed nor could be accounted for in analyses.

Second, although this study focused heavily on select $\mathrm{SDoH}$ as main predictors, these data required averaging/ summarizing across entire MSAs rather than being used at their collected level (eg, county and census tract). As a result, the values used may not have been an accurate representation of the entire MSA and may only have provided insight in urban areas, since non-MSA enrollees could not be matched by location to available $\mathrm{SDoH}$ data.

Third, only several SDoH factors were applied and there are likely a host of others that could be applied in a similar analysis. Future studies would benefit from examining additional SDoH factors, using data at a more granular level, and which may also be exact interpretations of the WHO matrix elements instead of suitable proxies.

Fourth, the data used were cross-sectional and could only account for events observed during the observation period; therefore, previous vaccination and/or events that may have affected what was sought during the current observation period that could have affected results were not able to be accounted for in these analyses.

Finally, results included only those with consistent health insurance coverage; consequently, results may not be generalizable to uninsured populations or those for whom cost may have been an impediment to vaccination. 


\section{Conclusions}

Vaccination against seasonal influenza remains suboptimal across the United States, and SDoH factors are likely contributors to variations in adults seeking immunization. The current study provides a strong rationale for further exploration of which elements of the SDoH framework play particularly important roles in vaccine acceptance and hesitancy. Common vaccine access points for adults, such as primary care clinics and community pharmacies, may be guided by these findings and should consider implementing strategies to improve vaccine uptake in their communities, particularly if their patient populations lack the ability to comprehend reliable health-related information. Similarly, managed care companies could consider using strategies to assist their members in seeking resources when their access to care changes and addressing the need for vaccination among those infrequently using their benefits.

\section{DISCLOSURES}

This study received no outside funding. Gatwood, Hagemann, Hohmeier, and Chiu declare vaccine-related grant funding from Merck \& Co. and GlaxoSmithKline for vaccine research unrelated to the current study. Ramachandran declares vaccine-related grant funding from GlaxoSmithKline for research unrelated to the current study. Shuvo and Behal have nothing to disclose.

Findings described in this study were presented as a poster and podium at the Academy of Managed Care Pharmacy Nexus 2020 Virtual meeting, October 19-23, 2020.

\section{REFERENCES}

1. Rolfes MA, Foppa IM, Garg S, et al. Annual estimates of the burden of seasonal influenza in the United States: a tool for strengthening influenza surveillance and preparedness. Influenza Other Respir Viruses. 2018;12(1):132-37.

2. Fine P, Eames K, Heymann D. Herd immunity: a rough guide. Clin Infect Dis. 2011;52(7):911-16.

3. Ozawa S, Portnoy A, Getaneh H, et al. Modeling the economic burden of adult vaccine-preventable diseases in the United States. Health Aff (Millwood). 2016; 35(11): 2124-32.

4. Centers for Disease Control and Prevention. Flu vaccination coverage, United States, 2018-19 influenza season. Accessed November 19, 2019. https:// www.cdc.gov/flu/fluvaxview/coverage1819estimates.htm

5. HealthyPeople.gov. Immunization and infectious diseases. Office of Disease Prevention and Health Promotion. Accessed November 19, 2019. https://www.healthypeople. gov/2020/topics-objectives/topic/ immunization-and-infectious-diseases/ objectives

6. Yang KC, Hung HF, Chen MK, et al. Cost-effectiveness analysis of universal influenza vaccination: application of the susceptible-infectious-complication-recovery model. Int J Infect Dis. 2018;73:102-08.

7. Centers for Disease Control and Prevention. Estimates of influenza vaccination coverage among adults-United States, 2017-18 flu season. Accessed November 19, 2019. https://www.cdc.gov/ flu/fluvaxview/coverage-1718estimates. $\underline{\text { htm }}$

8. MacDonald NE, SAGE Working Group on Vaccine Hesitancy. Vaccine hesitancy: definition, scope and determinants. Vaccine. 2015;33(34):4161-64.

9. Larson H, Jarrett C, Schulz W, et al. Measuring vaccine hesitancy: the development of a survey tool. Vaccine. 2015;33(34):4165-75.
10. World Health Organization. Social determinants of health. Accessed July 1, 2019. http://www.who.int/ social_determinants/en/

11. Pan American Health Organization. Social determinants of health in the Americas. Accessed July 23, 2019. https://www.paho.org/ salud-en-las-americas-2017/?p=45

12. World Health Organization. Closing the gap: policy into practice on social determinants of health. Discussion paper. 2011. Accessed July 23, 2019. https://apps. who.int/iris/handle/10665/44731

13. Larson HJ, Jarrett C, Eckersberger E, et al. Understanding vaccine hesitancy around vaccines and vaccination from a global perspective: a systematic review of published literature, 2007-2012. Vaccine. 2014;32(19):2150-59.

14. Butler R, MacDonald N, SAGE Working Group on Vaccine Hesitancy. Diagnosing the determinants of vaccine hesitancy in specific subgroups: The Guide to Tailoring Immunization Programmes (TIP). Vaccine. 2015;33(34):4176-79.

15. Mesch GS, Schwirian KP. Social and political determinants of vaccine hesitancy: lessons learned from the H1N1 pandemic of 2009-2010. Am J Infect Control. 2015;43(11):1161-65.

16. Freimuth VS, Jamison AM, An J, et al. Determinants of trust in the flu vaccine for African Americans and Whites. Soc Sci Med. 2017;193:70-79.

17. Nagata JM, Hernandez-Ramos I, Kurup AS, et al. Social determinants of health and seasonal influenza vaccination in adults $\geq 65$ years: a systematic review of qualitative and quantitative data. BMC Public Health. 2013;13(388):1-25.

18. Schmid P, Rauber D, Betsch C, et al. Barriers of influenza vaccination intention and behavior - a systematic review of influenza vaccine hesitancy, 2005-2016. PLoS One. 2016;12(1):1-46.

19. Centers for Disease Control and Prevention. Social determinants of health: know what affects health. Accessed March 17, 2021. https://www.cdc.gov/ socialdeterminants/index.htm 
20. National Health Literacy Mapping to Inform Health Care Policy. Health literacy data map. University of North Carolina at Chapel Hill. Accessed July 2, 2018. http:// healthliteracymap.unc.edu/

21. MIT Election Data and Science Lab. County presidential election returns 2000-2016. Massachusetts Institute of Technology. Accessed July 2, 2018. https://electionlab.mit.edu/

22. US Census Bureau. American Community Survey, 2014. Accessed July 2, 2018. https://factfinder.census.gov/

23. Comer KF, Grannis S, Dixon BE, Bodenhamer DJ, Wiehe SE. Incorporating geospatial capacity within clinical data systems to address social determinants of health. Public Health Rep. 2011;126 (Suppl 3):54-61.

24. Gatwood J, Shuvo S, Hohmeier KC, et al. Pneumococcal vaccination in older adults: an initial analysis of social determinants of health and vaccine-seeking behavior. Vaccine 2020;38(35):5607-17.

25. Shuvo S, Hagemann T, Hohmeier KC, Chiu CY, Ramachandran S, Gatwood J. The role of social determinants in timely herpes zoster vaccination among older American adults: a retrospective crosssectional study. Hum Vaccin Immunother. 2021;17(7):2043-49.

26. Gatwood J, Chiu CY, Shuvo S, et al. Role of social determinants of health in pneumococcal vaccination among highrisk adults. Vaccine. 2021;39(14):1951-62.

27. Office of Disease Prevention and Health Promotion. Healthy People 2020. Immunization and infectious diseases. U.S. Department of Health and Human Services. Accessed May 26, 2021. https://www.healthypeople. gov/2020/topics-objectives/topic/ immunization-and-infectious-diseases/ objectives
28. Office of Disease Prevention and Health Promotion. Healthy People 2030. Increase the proportion of people who get the flu vaccine every year-IID-09. U.S. Department of Health and Human Services. Accessed May 26, 2021. https://health.gov/healthypeople/ objectives-and-data/browse-objectives/ vaccination/increase-proportion-peoplewho-get-flu-vaccine-every-year-iid-09

29. Gatwood J, Meltzer MI, Messonnier M, Ortega-Sanchez IR, Balkrishnan R, Prosser LA. Seasonal influenza vaccination of healthy working-age adults: a review of economic evaluations. Drugs. 2012;72(1):35-48.

30. Drozd EM, Miller L, Johnsrud M. Impact of pharmacist immunization authority on seasonal influenza immunization rates across states. Clin Ther. 2017;39(8):1563-80.e17.

31. Centers for Disease Control and Prevention. Influenza vaccinations administered to adults in pharmacies and physician medical offices, United States. 2021. Accessed May 26, 2021. https:// www.cdc.gov/flu/fluvaxview/dashboard/ vaccination-administered.html

32. Centers for Disease Control and Prevention. Weekly US map: influenza summary update. Accessed May 26. 2021. https://www.cdc.gov/flu/weekly/usmap. $\underline{\mathrm{htm}}$

33. Centers for Medicare and Medicaid Services, Office of Minority Health. Annual influenza vaccination disparities in Medicare beneficiaries. Data Snapshot. November 2021. Accessed May 26, 2021. https://www.cms.gov/files/document/ data-snapshot-annual-influenza-vaccination.pdf.

34. Cutrona SL, Golden JG, Goff SL, et al. Improving rates of outpatient influenza vaccination through EHR portal messages and interactive automated calls: a randomized controlled trial. J Gen Intern Med. 2018;33(5):659-67.
35. Isenor JE, Edwards NT, Alla TA, et al. Impact of pharmacists as immunizers on vaccination rates: a systematic review and meta-analysis. Vaccine. 2016;34(47):5708-23.

36. Terrell-Perica SM, Effler PV, Houck PM, Lee L, Crosthwaite GH. The effect of a combined influenza/ pneumococcal immunization reminder letter. Am J Prev Med. 2001;21(4):256-60.

37. Tian S, Wang H, Wang W, Luo X. Characteristics associated with influenza vaccination among adults. J Public Health. 2019;41(3):e267-e273.

38. Lucyk K, Simmonds KA, Lorenzetti DL, et al. The association between influenza vaccination and socioeconomic status in high income countries varies by the measure used: a systematic review. BMC Med Res Methodol. 2019;19(1):153.

39. Lorini C, Santomauro F, Donzellini $\mathrm{M}$, et al. Health literacy and vaccination: a systematic review. Hum Vaccin Immunother. 2018;14(2):478-88.

40. Lu H, Winneg K, Jamieson KH, et al. Intentions to seek information about the influenza vaccine: the role of informational subjective norms, anticipated and experienced affect, and information insufficiency among vaccinated and unvaccinated people. Risk Anal. 2020;40(10):2040-56.

41. Igoe KJ. Establishing the truth: Vaccines, social media, and the spread of misinformation. Harvard T.H. Chan School of Public Health. July 10, 2019. Accessed May 26, 2021. https://www.hsph.harvard. edu/ecpe/vaccines-social-media-spreadmisinformation/

42. Wilson SL, Wiysonge C. Social media and vaccine hesitancy. BMJ Glob Health. 2020;5(10):e004206. 